\title{
Cross-Layer Effects on Training Neural Algorithms for Video Streaming
}

\author{
Pablo Gil Pereira \\ Saarland Informatics Campus \\ Saarbrücken 66123 \\ gilpereira@cs.uni-saarland.de
}

\author{
Andreas Schmidt \\ Saarland Informatics Campus \\ Saarbrücken 66123 \\ andreas.schmidt@cs.uni-saarland.de
}

\author{
Thorsten Herfet \\ Saarland Informatics Campus \\ Saarbrücken 66123 \\ herfet@cs.uni-saarland.de
}

\begin{abstract}
Nowadays Dynamic Adaptive Streaming over HTTP (DASH) is the most prevalent solution on the Internet for multimedia streaming and responsible for the majority of global traffic. DASH uses adaptive bit rate (ABR) algorithms, which select the video quality considering performance metrics such as throughput and playout buffer level. Pensieve is a system that allows to train ABR algorithms using reinforcement learning within a simulated network environment and is outperforming existing approaches in terms of achieved performance. In this paper, we demonstrate that the performance of the trained ABR algorithms depends on the implementation of the simulated environment used to train the neural network. We also show that the used congestion control algorithm impacts the algorithms' performance due to cross-layer effects.
\end{abstract}

\section{CCS CONCEPTS}

-Networks $\rightarrow$ Network control algorithms; Network experimentation; Network measurement; -Information systems $\rightarrow$ Multimedia streaming;

\section{KEYWORDS}

dynamic adaptive streaming, cross-layer effects, congestion control

\section{ACM Reference format:}

Pablo Gil Pereira, Andreas Schmidt, and Thorsten Herfet. 2018. Cross-Layer Effects on Training

Neural Algorithms for Video Streaming. In Proceedings of 28th ACM SIGMM Workshop on Network and Operating Systems Support for Digital Audio and Video, Amsterdam, Netherlands, fune 12-15, 2018 (NOSSDAV'18), 6 pages. DOI: $10.1145 / 3210445.3210453$

\section{INTRODUCTION}

Considering its share of global bandwidth usage, video streaming is the most important Internet application nowadays, and is predicted to increase even more in the next years [5]. These overthe-top (OTT) services employ adaptive bit rate (ABR) algorithms to adapt the video download to the current network state, considering parameters such as the estimated throughput or buffer occupancy level. When selecting the next bit rate, the goal is to maximize the

Permission to make digital or hard copies of all or part of this work for personal or classroom use is granted without fee provided that copies are not made or distributed for profit or commercial advantage and that copies bear this notice and the full citation on the first page. Copyrights for components of this work owned by others than ACM must be honored. Abstracting with credit is permitted. To copy otherwise, or republish, to post on servers or to redistribute to lists, requires prior specific permission and/or a fee. Request permissions from permissions@acm.org.

NOSSDAV'18, Amsterdam, Netherlands

(C) 2018 ACM. 978-1-4503-5772-2/18/06 ..\$15.00

DOI: $10.1145 / 3210445.3210453$ user's Quality of Experience (QoE) which depends on features such as startup delay, number of rebuffering events and visual quality that is dependent on the used bit rate and bit rate changes. Until recently, these ABR algorithms were designed with a specific QoE metric in mind and hence were not usable across different use cases, for instance on-demand streaming and low-latency applications.

Pensieve [10] is the first solution training an ABR algorithm using Reinforcement Learning (RL). These ABR algorithms can adapt to different applications and user preferences by modifying the reward function that incorporates a QoE metric. While optimizing the model for perceived performance, Pensieve can learn an optimal ABR algorithm for different network conditions.

As an application layer protocol, DASH performance is not only dependent on the ABR algorithm, but also on its interaction with the lower layers, in particular HTTP and TCP. These cross-layer effects were first discovered by $[6,8]$. While the usage of HTTP has an influence on the performance, this is out of scope for this paper. Instead, the cross-layer analysis focuses on the TCP protocol, as TCP's Congestion Control Algorithms (CCAs) [2] determine the portion of available throughput the application can use. The crosslayer information has also been used lately to model QoE [14] and design ABR algorithms [16]. Given that CCAs provide different performance to DASH applications, Pensieve can consider the crosslayer effects and learn a different $\mathrm{ABR}$ algorithm for each of them.

The contribution of this paper is twofold: First, we extend Pensieve's network simulator to faithfully handle propagation roundtrip time. Second, we evaluate the cross-layer dependencies between channel characteristics, TCP congestion control algorithms, QoE metrics, and the resulting trained ABR algorithms.

The rest of the paper is structured as follows. In Sec. 2, we describe how ABR algorithms are learned by Pensieve and the CCAs that are used for the evaluation. Modifications we applied to Pensieve are presented in Sec. 3 and evaluated in Sec. 4. We compare our work to other efforts in the area of cross-layer analysis of DASH performance in Sec. 5. Finally, we give directions for future research in Sec. 6 and conclude the paper in Sec. 7.

\section{ADAPTIVE VIDEO STREAMING SYSTEMS}

From a high level, the performance of a DASH system is measured based on the quality of the video that is received. This is influenced by the ABR algorithm, chosing an appropriate quality to request and taking the buffer occupancy as well as the throughput into account. The latter is determined by TCP's congestion control, which itself runs a control loop to probe for available bandwidth and get a fair share. Consequently, there are two layers with control loops that are usually independent by design, but entangled at runtime [8]. 


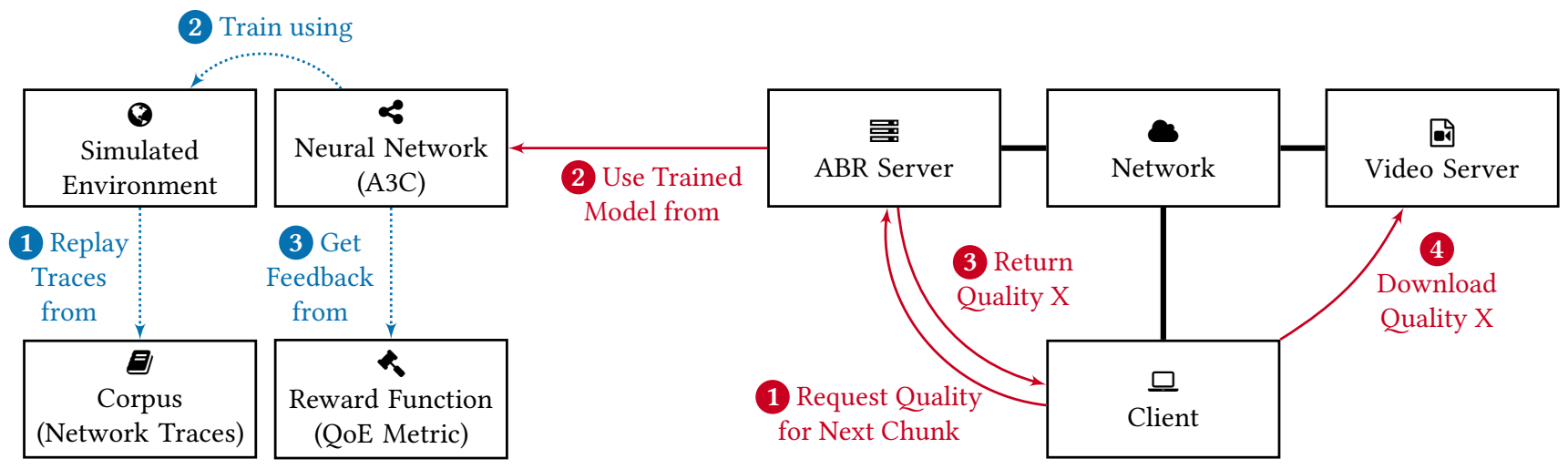

Figure 1: Pensieve's process is separated into the Training (blue dotted) and Execution (red solid) phases, which share the trained model for adaptive streaming.

\subsection{ABR Algorithms and Pensieve}

Research in the area of DASH has yielded many different ABR algorithms [12, 13, 16, 17] as well as QoE metrics [1,9]. Each ABR algorithm is designed to maximize the value of a specific QoE metric, so that in principle we have a direct relation between $A B R$ algorithm and QoE metric. As soon as a new QoE metric is defined or an existing one is modified, the ABR algorithm must be updated, which might require major changes in the bit rate selection strategy.

Pensieve [10] is the first solution that trains an ABR algorithm with network data and uses a given QoE metric to guide the training. The process is depicted in the left part of Fig. 1 and uses reinforcement learning to train with a specific data set, i.e. a single video with different bit rates. The learning is done by an A3C network [11], which uses asynchronous gradient descent to train neural networks and provides a policy vector as output. The training input includes information on throughput measurements, current buffer level, and more parameters. The training uses a corpus of network traces that are composed of throughput samples over time, together with a simulator that faithfully models a network with the respective throughput characteristics. In Sec. 3.1, these traces are extended by a further dimension to increase the fidelity of the simulator. The rewards are provided to the algorithm by evaluating a QoE metric, hence measuring the performance of taking a given action and guiding the algorithm to maximize the value.

After the training, the neural network is used inside the ABR server depicted in Fig. 1. Before requesting a chunk, the client queries the ABR server and receives the next quality to be downloaded. Although they can run on different systems for scalability reasons, we run the client and the $\mathrm{ABR}$ server on the same system.

In the following, we use Pensieve in its original as well as a modified version to learn ABR algorithms and afterwards evaluate them using the Pensieve DASH client and ABR server.

\subsection{Congestion Control Algorithms}

Over the last decades, many different CCAs have been developed with the goal of improving stability, fairness, and utilization of TCP connections over the Internet. The most recent standardized version is in RFC 5681 [2] and for instance Linux is using CUBIC [7]. These solutions consider a loss due to retransmission timout as a signal for congestion and reduce their throughput.

BBR [4] instead uses measurements of the round-trip propagation delay (RTprop) and the bottleneck bandwidth (BtlBw) to operate at the bandwidth-delay product $(B D P)$, which ensures that the throughput is maximized while the delay is minimized. As congestion causes queuing delays that result in an increase of RTT compared to RTprop, this is used as a signal that congestion is happening and causes BBR to reduce its sending rate.

In our evaluation, we run different experiments that use either CUBIC or BBR and show which effects the selection of a specific CCA can have on the overall DASH performance in terms of QoE.

\section{EVOLVING PENSIEVE}

Pensieve outperforms existing ABR algorithms regarding the QoE achieved in the wild [10]. We found that this performance can be improved by modifying the network simulator and extending the network corpus used for the training.

\subsection{Propagation Delays in the Simulator}

While Pensieve uses traces with samples of throughput over time, it does not incorporate the round-trip time and in particular the propagation delay that is independent of the throughput. One of the major differences between CUBIC and BBR is how they manage their congestion window, which has an impact on the portion of the RTT that is caused by the queuing delay.

3.1.1 Timed Traces. Following the data-driven learning approach, it is thus straightforward to extend the traces in a backwardscompatible way by adding a column that captures the round-trip propagation time. Consequently, for traces $i=0, \ldots, n-1$, we store:

- $t_{i},\left[t_{i}\right]=$ second: Relative time to the start of the trace.

- $b w_{i},\left[b w_{i}\right]=\frac{M b i t}{\text { second }}$ : Throughput measured at $t_{i}$.

- $R T$ prop $_{i},[$ RT prop $i]=$ second: Round-trip propagation time measured at $t_{i}$. 
Cross-Layer Effects on Training

Neural Algorithms for Video Streaming

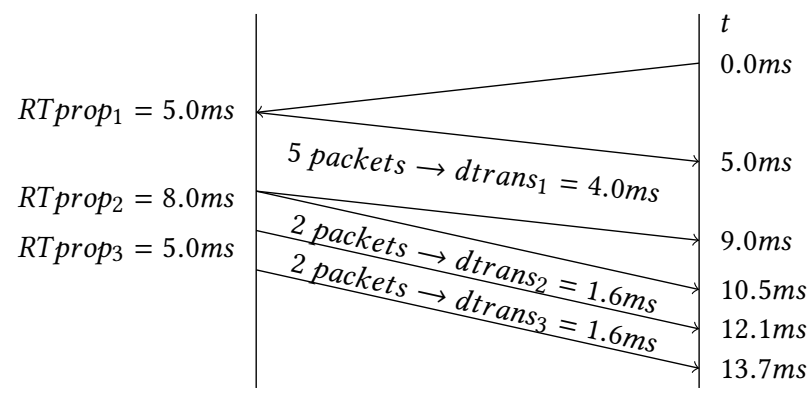

Figure 2: Model of overall delays with varying propagation delays for packets of 1500 bytes sent at 15Mbps.

3.1.2 Pensieve's Original Simulator (SIM-O). The original simulator in Pensieve, referred to as SIM-O in the following, uses a fixed round-trip propagation delay of $80 \mathrm{~ms}$, which is added to the transmission delay of a video chunk. This transmission delay depends on the throughput samples in the trace. After the propagation and transmission delay are summed up, SIM-O adds $\pm 10 \%$ to the total delay, causing uniformly distributed noise. Consequently, the randomness the simulation faces is proportional to the propagation delay as well as the transmission delay, so that the transmission times are also noisy. While propagation delay can vary in the wild, e.g. as a network card does not have a steady data rate, this additional noise is high. DASH segments of length $4 s$ and encoded at $4 \mathrm{Mbps}$ take $1 s$ at $16 \mathrm{Mbps}$ line rate of a residential connection. Adding $10 \%$ of noise would result in more than doubling the original delay of $80 \mathrm{~ms}$. As the variations in line-rate are already part of the throughput traces, this approach adds this variation twice.

3.1.3 Modified Simulator with Timed Traces (SIM-T). In order to investigate the impact of propagation delay handling within the simulator, we first augmented the traces, which resulted in a structure as in Sec. 3.1.1. We used the corpus described in Sec. 3.2.2 which uses the same base RTprop as the original simulator.

The RTprop a complete chunk faces is computed as the propagation time for the request and the maximum of propagation delays faced by the responses. This is further motivated by the exemplary transmission in Fig. 2, where an initial RTprop of $5 \mathrm{~ms}$ increases to $8 \mathrm{~ms}$ and returns back to $5 \mathrm{~ms}$ after a handful of packets. The transmission delay of the chunk is the sum of transmission delays for the individual packets, which vary with the throughput from the trace. Hence, we get the following delays for chunk $i$ which was transmitted using packets $p=0, \ldots, n-1$, each of size $P$ :

$$
\begin{gathered}
R T \operatorname{Trop}(i)=\frac{R T \operatorname{Rrop}_{0}}{2}+\max _{p=0, \ldots, n-1}\left(\frac{R T \operatorname{Trop}_{p}}{2}\right) \\
\operatorname{dtrans}(i)=\sum_{p=0}^{n-1} \frac{P}{b w_{p}}
\end{gathered}
$$

While this model does not consider reordering of packets in a flow, e.g. by route changes, it models the actual behavior of a channel more closely and still keeps the simulation straightforward. Assuming the client selects a bit rate roughly the same as the fair
NOSSDAV'18, June 12-15, 2018, Amsterdam, Netherlands

share, a chunk download takes the length of the chunk in seconds, which is of an order of magnitude smaller than the route change period, so that we can assume the route stays constant.

\subsection{Network Trace Corpora}

We have used different corpora of network traces for the evaluations in Sec. 4. Firstly, the Pensieve corpus [10] and variations of it, which were created using publicly available datasets. Secondly, what we call the DASH corpora, as they have been generated using traces from DASH-IF clients running on the testbed introduced in Sec. 4.1.2. Each corpus is separated into training and test sets to avoid overfitting and evaluate the performance of resulting algorithms on data they have not processed before.

3.2.1 Pensieve Corpus. This is the corpus used in [10], in the following referred to as $C_{P}$. Two public datasets were used to compose it with broadband and mobile Internet traffic, respectively. The traces are taken from the "web browsing" category in both datasets, meaning only HTTP downloads are considered, which range from regular, static web pages to streamed video.

3.2.2 Pensieve with RTprop Corpus. In order to incorporate the propagation delay, we have augmented the original traces with a base $R T$ prop of $80 \mathrm{~ms}$ and added $\pm 10 \%$ uniformly distributed random noise, which yields the corpus $C_{R T}$ prop. Considering the original implementation of the simulator, this is the corpus to be used to mimic the original behavior of Pensieve as closely as possible.

3.2.3 DASH Corpora. Several CCAs are currently available, which achieve different performance with DASH, as shown in [14] and analyzed in Sec. 4.4. In order to evaluate whether Pensieve can circumvent cross-layer effects, we ensure that only the performance achieved by a single CCA is represented in the traces. Two DASH corpora are generated complying with these requirements, namely $C_{C U B I C}$ and $C_{B B R}$, which are generated only considering traces collected with CUBIC and BBR, respectively.

These traces are collected in a controlled environment, which is described in Sec. 4.1.2. In order to avoid overfitting for a single throughput, we have configured the link with $\{3,3.5,4,4.5\} \mathrm{Mbps}$, while the latency is always $80 \mathrm{~ms}$. These corpora are generated just considering throughput and latency samples achieved by the DASH client, as DASH has an on-off traffic pattern whose performance differs from other applications [8]. The traces were collected 25 times for periods of 5 minutes.

We assume there is a correlation between consecutive throughput or latency traces within a download. For instance, if the congestion window is increased beyond the channel's BDP, that would result in a higher share of the available throughput, but this results in a higher RTT because of a queueing delay at the bottleneck buffer. Therefore, the traces in these corpora are not randomly selected, because this removes the correlation information and results in pathological, unrealistic network behaviours. In order to keep this information, the traces are collected in the sequence they were originally captured within the video download, with 1s granularity.

Despite not being collected in a realistic environment, the DASH corpora fulfill their purpose of representing the performance DASH applications achieve depending on the underlaying congestion control. Given that BBR and CUBIC also achieve different performance 
in the wild [4], we think the results in Sec. 4.4 also hold for traces collected in a more realistic scenario. In Sec. 6 we mention methods to collect realistic traces sorted by CCA.

\subsection{Entropy Weights and Training Duration}

Pensieve uses the gradient of the entropy of the policy vector as regularization term to avoid overfitting. The weight of the regularization term is a major hyperparameter for the learning phase, as the model tends to prematurely converge to suboptimal policies at the beginning of the training. The authors in [10] suggest starting with a high entropy factor of approx. 1.0 and progressively reduce it to 0.1 over 50,000 iterations. However, they do not specify how the entropy factor is changed over time. Having tested stepwise and linear approaches, we get the best performance when the models are trained with linear decrease from 1.0 to 0.1 with steps of 0.01 over 100,000 iterations. Thus, all the models presented in this paper have been trained with this policy. The selection of the optimal number of iterations and entropy weight progression is out of scope for this paper, but we are confident that finding such a scheme can also improve what our models can achieve.

\section{EVALUATION}

With the changes applied to Pensieve, we evaluate the resulting performance and consider the cross-layer effects that the choice of a given QoE metric and CCA have on the overall performance.

\subsection{Methodology}

For the experiments, we compare different QoE metrics, use different network scenarios and characteristics, and employ the dataset from the Pensieve paper.

4.1.1 QoE Metrics. In order to measure the performance of the neural network we use the general QoE metric defined in Eq. 3 for our experiments [17]. For a total number of $N$ chunks, the metric considers each chunk's bitrate $R_{n}$ and rebuffering time $T_{n}$, which results from the download of that chunk. The function $q\left(R_{n}\right)$ is the bitrate utility that maps the bitrate $R_{n}$ to the quality perceived by the user. The last term penalizes video quality switches to favor smoothness. Finally, $\mu$ is the rebuffering penalization term.

$$
Q o E=\sum_{n=1}^{N} q\left(R_{n}\right)-\mu \sum_{n=1}^{N} T_{n}-\sum_{n=1}^{N-1}\left|q\left(R_{n+1}\right)-q\left(R_{n}\right)\right|
$$

We have used two different bit rate utility functions, resulting in the following two QoE metrics:

- $Q o E_{l i n}$ : Linear mapping, where the utility function is the chunk's bit rate. Therefore, we set $q\left(R_{n}\right)=R_{n}$ and $\mu=4.3$ as in [17].

- $Q o E_{H D}$ : This metric assigns higher values to high definition (HD) video than it does for lower qualities. The bit rate-to-quality mapping for the Pensieve dataset can be found in Tab. 1 and sets $\mu=8$ as in [10].

\begin{tabular}{|c|c|c|c|c|c|c|}
\hline$R_{n}(M b p s)$ & 0.3 & 0.75 & 1.2 & 1.85 & 2.85 & 4.3 \\
\hline$q\left(R_{n}\right)$ & 1 & 2 & 3 & 12 & 15 & 20 \\
\hline
\end{tabular}

Table 1: Mapping of $\mathrm{QoE}_{\mathrm{HD}}$ bit rates to quality levels.

4.1.2 Scenario. The testbed uses IPSec and L2TP tunnels through the GEANT ${ }^{1}$ research network for connecting the two remote locations and OpenvSwitch to do the bridging. All hosts are VirtualBox virtual machines (VM) running the same version of Ubuntu and they are connected to the nodes via VirtualBox host-only interfaces, which are then bridged to OpenvSwitch. Across the L2TP tunnel we apply network emulation (netem) to add delays to achieve a total round-trip time of $80 \mathrm{~ms}$, as well as for packet loss emulation for Sec. 4.4.1, and token bucket filter (tbf) to limit the throughput to different values.

4.1.3 Dataset. For all the experiments we have used the same video dataset used in [10], which is encoded by the H.264/MPEG-4 codec at the bitrates $\{0.3,0.75,1.2,1.85,2.85,4.3\} \mathrm{Mbps}$. The video, which has a total lenght of 193 seconds, has been divided into 48 chunks of 4 seconds each and a last chunk of 1 second.

\subsection{Implementation}

The experiment has three separate components, namely the client, the ABR server and the DASH server. While the DASH server runs in a dedicated VM, the DASH client and ABR server run on the same VM. The operating system in all the VMs is Ubuntu 16.04 LTS with Linux kernel 4.9, which is the first kernel including BBRv1.0 [4].

The DASH server is an Apache server v2.4.18 with the crossorigin resource sharing (CORS) capabilities enabled. The ABR server is presented in [10], which uses BaseHTTPServer ${ }^{2}$ to handle HTTP requests from the client and TensorFlow to execute the Actor network. Finally, we used the DASH-IF client provided in the Pensieve repository $^{3}$, which requests the next bitrate to the $A B R$ server instead of using the default offline ABR algorithm [10].

\subsection{SIm-O vs. Sim-T}

In order to show the impact of propagation delay handling, we compared the performance of ABR algorithms trained using Pensieve's original simulator (SIM-O) and those trained using our simulator with timed traces (SIM-T), which were presented in Sec. 3.1. For both simulators we used $Q o E_{H D}$, which is an aggressive metric. For this experiment we use the testbed described in Sec. 4.1.2 with $80 \mathrm{~ms}$ RTT and two throughput configurations: a limited scenario with $3 \mathrm{Mbps}$, where the available throughput is smaller than the highest video bit rate, and an unlimited scenario with $6 \mathrm{Mbps}$. The QoE samples have been collected for 5 minutes for each execution.

The models resulting from training with $Q o E_{H D}$ have a better performance when trained with SIM-T instead of Sim-O (Fig. 3). Therefore, the proper handling of RTTs in the simulator allows more aggressive decisions, which in turn results in better user experience. ABR algorithms learned using SIM-T are trained in a more faithful streaming environment, which leads to a situation where it can

\footnotetext{
${ }^{1}$ https://www.geant.org/

${ }^{2}$ https://docs.python.org/2/library/basehttpserver.html

${ }^{3}$ https://github.com/hongzimao/pensieve
} 
Cross-Layer Effects on Training

Neural Algorithms for Video Streaming
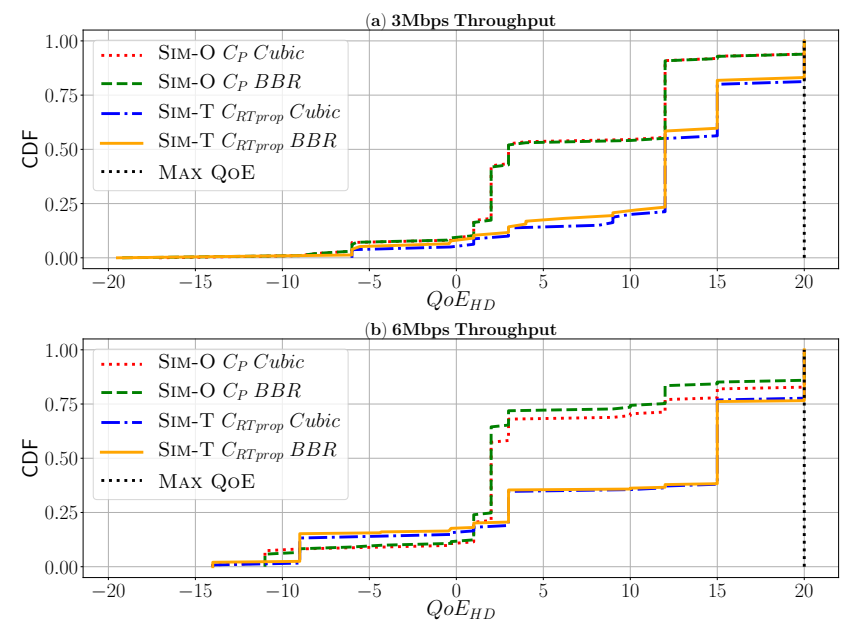

Figure 3: Comparison of SIM-O and SIM-T with $Q o E_{H D}$ and bottleneck bandwidth of $3 \mathrm{Mbps}$ and $6 \mathrm{Mbps}$.
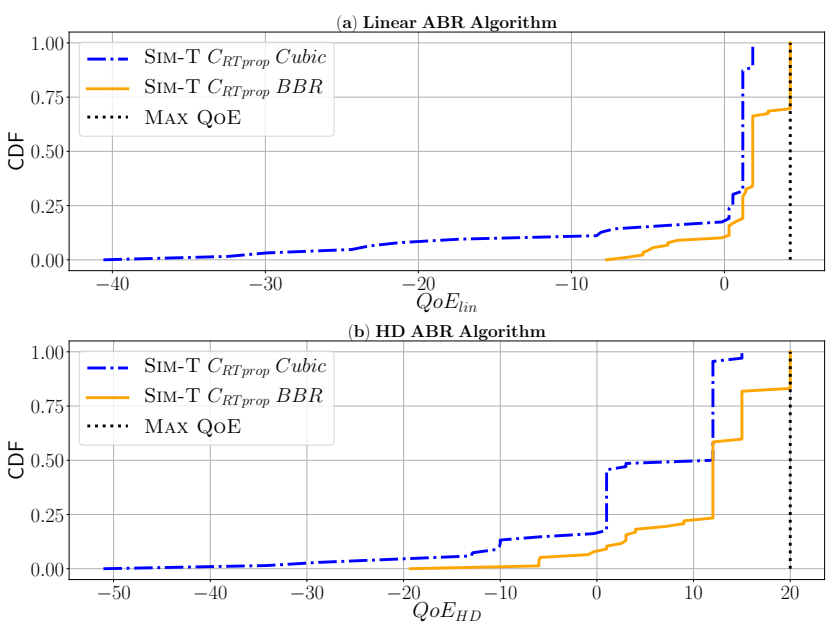

Figure 4: Scenarios with 2\% loss lead to significant difference in the performance of $\mathrm{ABR}$ algorithms, depending on the used CCA and QoE metric.

make better decisions than those that use Sim-O. It is important to note that this performance difference is only due to a change in the simulator and without any changes in the neural network used to learn the $\mathrm{ABR}$ algorithm.

\subsection{Cross-layer Effects with Pensieve}

Previous work shows cross-layer effects on DASH depending on the TCP layer, so we are going to investigate the effects of using different TCP algorithms on the achieved QoE for learned ABR algorithms. We train ABR algorithms on corpora that used a single CCA and show that this has an impact on the resulting performance.

4.4.1 Channel Loss. A major difference between BBR and CUBIC is the throughput they can sustain in scenarios with significant
NOSSDAV'18, June 12-15, 2018, Amsterdam, Netherlands

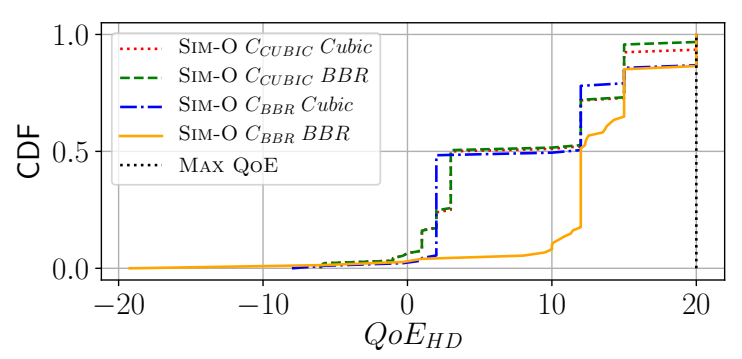

Figure 5: Comparison of ABR algorithms trained on CUBIC and BBR DASH corpora and executed with CUBIC and BBR indicate cross-layer effects with neural adaptive streaming.

channel loss, as shown in [4]. For evaluating its impact on DASH applications, we compare how models trained with SIM-T perform with $2 \%$ packet loss. We omit Sim-O in this analysis as Sec. 4.3 shows that the training with SIM-T results in better performance.

We can see in Fig. 4 that DASH achieves better performance using BBR as the CCA, independently of the used QoE metric. This is due to the stable throughput BBR can achieve on lossy channels, as it does not reduce the congestion window with a packet loss. There is a major gap between CUBIC and BBR with $Q o E_{H D}$. This is in line with the results in [8], which show that DASH needs to choose bit rates aggressively to allow TCP to effectively probe for the available throughput. This is the case for $Q o E_{H D}$, which selects higher bit rates leading to higher rewards during training.

4.4.2 Congestion Control Algorithm. Using the $C_{C U B I C}$ and $C_{B B R}$ corpora, which are described in Sec. 3.2 .3 , we trained two instances of SIM-O to compare the performance of the resulting algorithms. The scenario in Sec. 4.1.2 is configured with $3 \mathrm{Mbps}$ throughput, $80 \mathrm{~ms}$ RTT, and no loss.

Fig. 5 shows that the model with the best performance is trained with $C_{B B R}$ and executed with BBR. As BBR aims to operate at the $\mathrm{BDP}$, it can achieve a more constant throughput, as the congestion window is not reduced with losses caused by bandwidth probing. SIM-O with $C_{C U B I C}$ executed with BBR is not able to exploit BBR's desirable characteristic, as the model learned to run on top of CUBIC, resulting in the CUBIC-like performance. Fig. 5 also shows that a mismatch of the CCA between traces collection and client execution might lead to poor performance.

In consequence, DASH performance depends on the underlying $\mathrm{CCA}$ as Pensieve learned a different $\mathrm{ABR}$ algorithm for BBR and CUBIC, so cross-layer effects have to be considered. As a result of their impact in the performance of the learned ABR, we think CCAs should be considerend for a more realistic traces collection.

\section{RELATED WORK}

Cross-layer effects for HTTP Adaptive Streaming (HAS), were first discovered by Esteban et al. [6], who describe the effects during the different phases of TCP congestion control, namely initial burst, ACK clocking and trailing ACK phases. They show that loss is most damaging in the trailing ACK phase, where packets are still in flight but no new data can be sent. They also analyze how pacing avoids burst losses, but report only minimal throughput gains. 
Cross-layers effects also impact the design of ABR algorithms, as introduced by Huang et al. [8]. They point out that DASH "on-off" traffic scheduling prevents TCP for probing for its fair share. Thus, ABR algorithms should not rely on throughput estimation to select the bit rate. This is further investigated in Wang et al. [16], where an ABR algorithm based on cross-layer effects is presented.

The extensive emulation and analysis of cross-layer dependencies by Stohr et al. [14] found that there is a linear relation between delay, loss and QoE, that is independent of the bandwidth - a result that is further supported by our findings regarding the incorporation of latency information into the training. As a consequence, a corpus for Pensieve considering more features than only throughput is necessary, as well as changes in the neural network's input.

Another analysis of cross-layer behavior by Bhat et al. [3] investigates how replacing the TCP and HTTP layer within DASH can change the overall performance of DASH, which was also investigated by Timmerer et al. [15]. The authors show that unmodified DASH clients are not able to improve QoE when compared to DASH over TCP and even result in lower bit rates.

\section{FUTURE WORK}

While Pensieve can learn ABR algorithms that outperform existing ones, there are opportunities for future extensions beyond what the original paper describes.

Propagation Delay as Model Input. We have shown how important the propagation delay is for proper simulation of a network environment. We propose to extend the state vector Pensieve uses by measurements of the current round-trip propagation time. This RTT information becomes even more important when buffer size and chunk sizes shrink, which is the case for low-latency applications and hardware that incorporates a fixed amount of memory.

Leveraging (More) Cross-Layer Information. As Huang et al. [8] show, throughput should be measured by TCP itself and not within DASH. The same holds for other parameters such as losses or propagation delay, which can be measured by TCP implementations and used by the model as additional state information features. BBR for instance has a faithful model of the channel that can be used by the ABR algorithm for a better bit rate selection.

Extended Network Corpus. We think that actual network measurements for HTTP traffic that incorporate delay information are required, as well as traces sorted by CCA as shown in Sec. 4.4.2. Gathering this corpus could for instance be done using the RIPE Atlas ${ }^{4}$ project that allows to run HTTP measurements from a wide variety of end-systems to well-defined IP addresses or hostnames.

Reinforcement Learning for Low-latency Streaming. Pensieve, like most of the ABR algorithms, relies on a large playback buffer to overcome TCP throughput fluctuations. However, large buffers are not suitable for live streaming, since the video is not completely available in advance. In scenarios with such low-latency constraints, the buffer level becomes more important, as small fluctuations may result in re-bufferings. We suggest extending the state vector so that not only the last buffer level sample, but a short history of it is sent to the ABR server, which would allow to track how the application reads the buffer.

\section{CONCLUSION}

Training ABR algorithms for DASH using neural networks proves to yield outstanding results. Nevertheless, this performance can be improved even further by making the simulator used during training more realistic. As we have shown, replacing a randomized treatment of link round-trip times with a trace-based approach can help to achieve better results. We have also shown in our cross-layer analysis how the CCA algorithm impacts the performance of ABR algorithms. Finally, we predict that extending the training model by additional input signals and gather these signal from the transport layer can lead to a further increase of reliability and performance of neural adaptive streaming systems.

\section{ACKNOWLEDGMENTS}

The work is supported by the German Research Foundation (DFG) as part of SPP 1914 "Cyber-Physical Networking" under grant HE 2584/4-1.

\section{REFERENCES}

[1] Claudio Alberti, Daniele Renzi, Christian Timmerer, and Christopher Mueller. Automated QoE Evaluation of Dynamic Adaptive Streaming Over HTTP. 5th International Workshop on Quality of Multimedia Experience (QoMEX), 2013.

[2] M. Allman, V. Paxson, and E. Blanton. TCP Congestion Control. RFC 5681 (Draft Standard), September 2009.

[3] Divyashri Bhat, Amr Rizk, and Michael Zink. Not so QUIC: A Performance Study of DASH over QUIC. In Proceedings of the 27th Workshop on Network and Operating Systems Support for Digital Audio and Video, pages 13--18. ACM, 2017.

[4] Neal Cardwell, Yuchung Cheng, C Stephen Gunn, Soheil Hassas Yeganeh, and Van Jacobson. BBR: Congestion-Based Congestion Control. ACM Queue, 14(5):50:2050:53, 2016.

[5] Cisco. The Zettabyte Era: Trends and Analysis. (June 2017):1-32, 2017.

[6] Jairo Esteban, Steven Benno, Andre Beck, Yang Guo, Volker Hilt, and Ivica Rimac. Interactions between HTTP adaptive streaming and TCP. Proceedings of the 22nd international workshop on Network and Operating System Support for Digital Audio and Video - NOSSDAV '12, pages 21-26, 2012.

[7] Sangtae Ha, Injong Rhee, and Lisong Xu. CUBIC : A New TCP-Friendly HighSpeed TCP Variant. ACM SIGOPS Operating Systems Review - Research and developments in the Linux kernel, 42(5):64-74, 2008.

[8] Te-Yuan Huang, Nikhil Handigol, Brandon Heller, Nick NcKeown, and Ramesh Johari. Confused, Timid, and Unstable: Picking a Video Streaming Rate is Hard. Proceedings of the ACM conference on Internet measurement, pages 225-238, 2012.

[9] Yao Liu, Sujit Dey, Fatih Ulupinar, Michael Luby, and Yinian Mao. Deriving and Validating User Experience Model for DASH Video Streaming. IEEE Transactions on Broadcasting, 61(4):651-665, 2015.

[10] Hongzi Mao, Ravi Netravali, and Mohammad Alizadeh. Neural Adaptive Video Streaming with Pensieve. In Proceedings of the Conference of the ACM Special Interest Group on Data Communication, pages 197-210. ACM, 2017.

[11] Volodymyr Mnih, Adria Puigdomenech Badia, Mehdi Mirza, Alex Graves, Timothy Lillicrap, Tim Harley, David Silver, and Koray Kavukcuoglu. Asynchronous methods for deep reinforcement learning. In International Conference on Machine Learning, pages 1928-1937, 2016.

[12] Yongtao Shuai and Thorsten Herfet. On stabilizing buffer dynamics for adaptive video streaming with a small buffering delay. In 14th IEEE Annual Consumer Communications \& Networking Conference (CCNC), pages 435-440. IEEE, 2017.

[13] Kevin Spiteri, Rahul Urgaonkar, and Ramesh K. Sitaraman. BOLA: Near-optimal bitrate adaptation for online videos. In Proceedings of the 35th Annual IEEE International Conference on Computer Communications, IEEE INFOCOM, 2016.

[14] Denny Stohr, Alexander Frömmgen, Jan Fornoff, Michael Zink, Alejandro Buchmann, and Wolfgang Effelsberg. QoE Analysis of DASH Cross-Layer Dependencies by Extensive Network Emulation. In Proceedings of the workshop on QoE-based Analysis and Management of Data Communication Networks, 2016.

[15] Christian Timmerer and Alan Bertoni. Advanced Transport Options for the Dynamic Adaptive Streaming over HTTP. arXiv preprint arXiv:1606.00264, 2016.

[16] Cong Wang, Amr Rizk, and Michael Zink. SQUAD: a spectrum-based quality adaptation for dynamic adaptive streaming over HTTP. In Proceedings of the 7th International Conference on Multimedia Systems, MMSys 2016, pages 1-12, 2016.

[17] Xiaoqi Yin, Abhishek Jindal, Vyas Sekar, and Bruno Sinopoli. A Control-Theoretic Approach for Dynamic Adaptive Video Streaming over HTTP. Proceedings of the 2015 ACM SIGCOMM, pages 325-338, 2015. 